\title{
Effect of Meal Frequency on the Loss Weight, Glycemia, Lipid Profile, Plasma Ghrelin and Energy Expenditure of Women with Obesity: A Clinical Trial
}

\author{
Érika Duarte Grangeiro ${ }^{1,}{ }^{*}$, Mariana Silva Trigueiro ${ }^{2}$, Leysimar de Oliveira Siais ${ }^{3}$, Hilana \\ Moreira Paiva ${ }^{4}$, Mauro Sola-Penna ${ }^{5}$ and Eliane Lopes Rosado ${ }^{6}$ \\ 1 Institute of Nutrition, Federal University of Rio de Janeiro; erikawduartegs@gmail.com \\ 2 Institute of Nutrition, Federal University of Rio de Janeiro; mariana19trigueiro@gmail.com \\ 3 Institute of Nutrition, Federal University of Rio de Janeiro; leysimarsiais.nut@gmail.com \\ 4 Institute of Nutrition, Federal University of Rio de Janeiro; hilana.paiva@hotmail.com \\ ${ }^{5}$ Laboratory of Enzymology and Metabolism Control, Federal University of Rio de Janeiro, Rio de Janeiro; \\ msolapenna@me.com \\ ${ }^{6}$ Institute of Nutrition, Federal University of Rio de Janeiro; elianerosado@nutricao.ufrj.br \\ * Correspondence: erikawduartegs@gmail.com; Carlos Chagas Filho Avenue, 373 - CCS - Block J - 2th Floor - \\ Cidade Universitária, Fundão Island, 21941-590 - Rio de Janeiro, RJ - Brazil
}

\begin{abstract}
Dietary approach is essential to obesity control, but the effectiveness of changes in meal frequency (MF) as strategies for loss and maintenance of body mass remain unclear. This study aimed to evaluate the influence of MF on a hypocaloric diet on weight loss, active ghrelin levels and metabolic indicators of women with obesity. This is a randomized, parallel clinical trial, including forty women, randomized in two groups, both following a hypocaloric diet, according to MF (G1 six meals/day; G2 - three meals/day). Dietary, laboratory, anthropometric and body composition indicators were assessed, as well as energy expenditure (EE), before and after the 90 days of intervention. After intervention, both groups decreased body weight, body mass index (BMI), waist circumference, fat mass (FM), insulin and HOMA-IR. G1 increased insulin sensitivity and G2 reduced triglyceride and FM and increased fat-free mass (FFM). MF increased ghrelin levels. There were no differences in EE variables. Hypocaloric diet with different MF promoted a reduction in total weight, BMI, WC and FM and an improvement in glycidic metabolism. However, the accomplishment of the three meals/day increased the FFM and active ghrelin and reduced triglyceride, while six meals/day was more beneficial in increasing insulin sensitivity.
\end{abstract}

Keywords: obesity; meal frequency; hypocaloric diet; energy expenditure; ghrelin; weight loss.

\section{Introduction}

Obesity is a chronic, multifactorial, complex and epidemic disease, of great importance to public health, reason that it can increase the risk of comorbidities including diabetes, systemic arterial hypertension, dyslipidemia, coronary heart diseases, gallstones, sleep apnea, other respiratory 
disorders and the incidence of certain types of cancer [1-3] According to the World Health Organization [3] estimative, there are over 1,9 billion of people over 18 years old with excess of body weight, including 650 million people with obesity in the world.

The obesity treatment must be multidisciplinary, consisting of reducing excessive body weight and instituting other measures to control the associated risk factors [4,5]. With regard to dietary treatment, the objective is to achieve a state of negative energy balance, by decreasing food consumption, since weight loss of $3 \%$ to $5 \%$, which is maintained, has the ability to produce clinically relevant changes $[4,6]$.

American Dietetic Association recognizes that respect for the influence of meal frequency (MF) on the application of total weight is limited, however, it is recommended that a total energy intake be distributed throughout the day, totalizing between five meals, including breakfast [7]. Currently, study of interventions suggests on the contrary to epidemiological studies, that the highest number of meals per day cannot improve more benefits for health and body weight control [8].

A relationship between different MF and body composition is pointed out in studies that showed that an increase (more than three meals per day) does not promote greater weight loss in individuals with obesity who consumed a hypocaloric diet [9-11]. It is questioned that eating more than three times per day may result in lower weight by increasing the thermal effect of food (TEF) and the fat oxidation [12].

Regarding the lipid profile, the outcomes suggest uncertainties in the literature about the ideal MF. Reduced meals per day (less than three meals per day) may indicate more favorable lipid profiles in individuals with obesity, however, it is seen in other studies that the profile of lipids and glycoregulatory factors (fasting glucose, insulin and insulin sensitivity) are not affected by changes in MF, suggesting that further investigations are needed [11,13]. Similarly, in the literature, the association between MF and satiety hormones is less consistent in obesity, however, it is reported that lower MF in a hypocaloric diet can have an effect on the best postprandial response of ghrelin $[14,15]$.

Developing a better understanding of the MF impact within obesity treatment, the purpose of this study was to examine the effect between different MF on weight loss, body composition, blood glucose, lipid profile, active ghrelin and energy expenditure (EE) outcomes among women with obesity enrolled in a weight loss intervention.

\section{Materials and Methods}

\subsection{Population}

Women with class I and II obesity, aged 20-45 years, from Rio de Janeiro, Brazil participated in this study. The eligible women were those of any ethnicity, not menopausal, without recent change in body weight ( $\pm 3 \mathrm{~kg}$ in the last 3 months) or on diets to lose weight in the last 3 months, as well as, those who have not undergone bariatric surgery. Athletes, users of medication or supplements for weight control, smokers, alcoholics, with cardiac and respiratory complications, clinical diagnosis of diabetes, decompensated hypothyroidism, pregnant, lactating, illiterates and those with inflammatory bowel disease, cancer, liver and severe kidney diseases were not eligible. In addition, women who suggested severe food compulsion, assessed by the Scale of Periodic Alimentary Compulsion (SPAC), were not selected.

Participants who did not comply with the proposed protocol during the intervention or who presented complications that interfered in their participation during the study were excluded. The recruitment was through the dissemination of the research on the page (online) at the Josué de Castro Nutrition Institute, as well as, through posters posted on the UFRJ campus.

This study was conducted in accordance with the Ethics and Research Committee of Hospital Clementino Fraga Filho under protocol CAAE 97290918.2.0000.5257, number 3.015.648 and published in the Brazilian Registry of Trials with the number RBR-7k6v3v. All volunteers signed an informed consent form. 


\subsection{Design}

This is a randomized and parallel clinical trial. The sample was for convenience. The first meeting aimed to check the eligibility criteria, apply SPAC, check body weight and calculate the body mass index (BMI). At the time, the women were clarified about how to fill the three-day dietary records (two typical and one atypical day) and the periodicals (in each re-consultation the participants were supposed to deliver one periodical, totaling three). Individuals were also advised regarding the preparation for blood collection, the evaluation of EE and body composition. Women were instructed not to change physical activities.

Participants were randomized into two groups: group 1 (hypocaloric diet + standard fractionation - six meals / day) (G1) and group 2 (hypocaloric diet + reduced fractionation - three meals / day) (G2). Randomization was performed through the website random.org. In the second meeting, the women went to collect blood sample, and then, variables anthropometrics, body composition and EE were assessed. The participants received a hypocaloric diet to be followed for 90 days and, a questionnaire was applied to evaluate the practice of physical activity.

Three individual meetings were carried out, until they completed the 12-week period, checking their body weight, waist circumference (WC), periodic food record, adherence to diet and clarifying doubts. Weekly contacts were made through messages or phone calls to check compliance with the food plan and clarify possible doubts during the research. After intervention, anthropometric, biochemical e dietetic and EE indicators were evaluated one more time, and the International Physical Activity Questionnaires (IPAQ) and the SPAC were filled out.

\subsection{Dietary intervention}

The dietary prescription was made by trained nutritionists and the diets using the software DietProClínico®6.1. The calculation of the hypocaloric diet was balanced and individualized, according to the resting metabolic rate (RMR) obtained by indirect calorimetry (IC), multiplying by the corresponding activity factor to obtain the total energy expenditure (TEE) [16]. Subsequently, it was diminished from the TEE $700 \mathrm{kcal} /$ day, to obtain a body weight reduction of approximately 2$4 \mathrm{Kg} /$ month.

The hypocaloric diet was recommended according to the reference dietary intake for carbohydrates ( 45 to $65 \%$ of TEE), lipids ( 20 to $35 \%$ of TEE) and proteins (15 to $35 \%$ of TEE) and dietary fiber did the Institute of Medicine (IOM) recommend prescribed (25 grams / day) for adult women [17]. The distribution of the lipid fractions were $<10 \%$ of the TEE for saturated fatty acids (SFA), 6 to $11 \%$ of the TEE of polyunsaturated fatty acids (PUFA), 15 to $20 \%$ of the TEE of monounsaturated fatty acids (MUFA) and cholesterol $<300 \mathrm{mg} /$ day $[18,19]$. The hypocaloric diet was fractionated into six daily meals (breakfast, snack, lunch, snack, dinner and supper) for G1 and three daily meals (breakfast, lunch and dinner) for G2.

\subsection{Dietary assessment, habitual physical activities and eating behavior}

All dietary registration forms were reviewed by dietitians in order to identify any omission about number of meals, added sugar, spoon size, amount consumed and type of preparation [20]. The chemical composition analysis (energy, proteins, carbohydrates, lipids, MUFA, PUFA, SFA, cholesterol and fibers) were performed in the DietProClínico ${ }^{6} 6.1$ diet analysis program. Information obtained in the dietary records were compared with the Dietary Reference Intake and the consumption of total fibers and fatty acids were compared with the IOM recommendations [17-19].

Adherence to the proposed diet plans was questioned, so that the participants self-reported, within a scale of 0 to $100 \%$, how much they were managing to comply with the stipulated meals and what obstacles they faced. IPAQ was used (short form), before and after the intervention, to characterize the population and to verify the maintenance of physical activity throughout the research [21]. SPAC was applied to observe the magnitude of eating behavior at the beginning and at the end of the study to assess whether there was any change during the intervention $[22,23]$. 


\subsection{Anthropometric characteristics, body composition and energy expenditure}

Body weight, WC and height were assessed in the morning while the participants were fasting, wearing light clothing and without shoes, using the electronic platform scale, flat steel anthropometric tape and a portable vertical anthropometer, respectively. WC was measured at the midpoint between the last cost and the iliac crest. Afterwards, BMI was calculated using the formula body mass (kg)/height (m2) and classified according to the recommendations by WHO [24].

Fat mass (FM) and fat-free mass (FFM) were estimated through bioelectrical impedance analysis (BIA) by Biodynamics $₫ 450$ and FFM was estimated using the predictive equation validated for women with obesity [25]. First the RMR was measured while each subject was lying down for 20 minutes, using CI (Vmax 29®, ViasysHealthcare, EUA).

All participants provided the conditions necessary for BIA and CI: normohydrates; 12 hours fast; no water intake for at least two hours; no alcohol, caffeine and excess of protein intake for 48-72 hours; no strenuous physical activity for 24 hours and during the follicular phase [25-28]. The RMR was calculated based on the results obtained in the CI assessment and estimated by equation of Weir [29].

\subsection{Laboratory analysis}

Blood samples were obtained from participants who were 12 hours overnight fasting for biochemical analysis and ghrelin. Lipid profiles (total cholesterol (TC), high-density lipoprotein cholesterol (HDL-c), LDL-c and triglycerides (TG)), glucose and insulin levels, these were analyzed for enzymatic UV test and insulin for chemiluminescence test [30-33]. LDL-C was estimated [34]. IR was calculated using the method HOMA-IR: fasting serum insulin concentration $(\mu \mathrm{U} / \mathrm{mL}) \times$ fasting blood glucose (mmol / L) / 22.5 and analyzed according to Stern et al. (2005) [35]. Insulin sensitivity (IS) was estimated using the Quantitative Insulin Sensitivity Check Index (QUICKI), QUICKI = 1 / (LOG (fasting serum insulin) + LOG (fasting blood glucose) [36].

The blood sample for ghrelin analysis was collected in tubes containing ethylenediamine tetraacetic acid (EDTA) and Pefabloc ${ }^{\circledR}$ (specific protein inhibitor). The analysis of active ghrelin was performed using the Enzyme Linked Immuno Sorbent Assay (Elisa) method using the commercial kit E-EL-H2002® (Elabscience, Inc, Wuhan, China).

\subsection{Statistical analysis}

All values were reported as the mean and standard deviation (SD) for Gaussian (normal) distribution and as median and quartile interval for non-Gaussian (non-normal) distribution. The Kolmogorov-Smirnov test was used adopting significance when $p<0.05$. Normally distributed indicators were assessed by the unpaired t test and comparisons of the baseline and after intervention in the same group were verified by the paired $t$ test. Mann Whitney test was applied for compare groups for non-normal distribution and Wilcoxon to analyze before and after intra-group intervention.

Spearman's correlation coefficient test was used to analyze the relationship between active ghrelin secretion and anthropometric indicators, suggestive of binge eating and dietary indicators. The statistical analysis program was SPSS (Statistical Package for Social Science, IBM Corporation, NY) version 22.0. In addition, the outlier test was performed for the results of active ghrelin using the Graphpad Prism 8.3 program.

\section{Results}

There were 283 women who showed interest in participating in the study, of these, 127 were not eligible, 105 gave up participating and 4 did not attend the contact. 47 women were randomized (G1: 24; G2: 23). In G1, 6 participants dropped out for personal reasons. In G2, one participant gave up and another was excluded because she got pregnant. Forty women with obesity participated in this study (G1:19; G2:21). Recruitment was conducted for 6 months. The study was not harmful for all participants. The groups showed similarities at baseline in the variables age, anthropometric 
indicators (weight, BMI and WC) and SPAC scores, however, insulin levels, HOMA-IR and TG were higher in G2 (Table 1). There was no difference in the practice of physical activity before $(p=0.16)$ and after $(p=0.21)$ intervention between the groups.

Table 1: Baseline Subjects Characteristics.

\begin{tabular}{|c|c|c|c|}
\hline & G1 $(n=19)$ & G2 $(n=21)$ & $p$-value \\
\hline Age (years) & $29.05 \pm 9.18$ & $30.33 \pm 6.72$ & 0.61 \\
\hline Weight (Kg) & $90.88 \pm 8.96$ & $92.96 \pm 11.09$ & 0.51 \\
\hline $\operatorname{IMC}\left(\mathrm{kg} / \mathbf{m}^{2}\right)$ & $34.90 \pm 14.65$ & $35.27 \pm 3.96$ & 0.76 \\
\hline $\mathrm{WC}(\mathrm{cm})$ & $96.94 \pm 8.88$ & $101.47 \pm 8.87$ & 0.11 \\
\hline FM (kg) & $41.99 \pm 6.45$ & $43.90 \pm 7.65$ & 0.39 \\
\hline FM (\%) & $46.02 \pm 3.58$ & $47.02 \pm 3.74$ & 0.39 \\
\hline RMR (kcal) & $1467.10 \pm 121.08$ & $1479.31 \pm 171.27$ & 0.79 \\
\hline Glucose (mg /dL) & $84.31 \pm 7.96$ & $92.38 \pm 18.24$ & 0.07 \\
\hline Insulin $(\mu \mathrm{U} / \mathrm{mL})$ & $17.63 \pm 8.06$ & $24.54 \pm 12.39$ & 0.04 \\
\hline HOMA-IR & $3.69 \pm 1.72$ & $5.55 \pm 2.72$ & 0.01 \\
\hline QUICKI & $1.53 \pm 0.16$ & $1.51 \pm 0.29$ & 0.78 \\
\hline $\mathrm{TC}(\mathrm{mg} / \mathrm{dL})$ & $169.52 \pm 35.49$ & $178.38 \pm 28.52$ & 0.39 \\
\hline HDL-c (mg /dL) & $51.21 \pm 13.06$ & $49.09 \pm 10.58$ & 0.58 \\
\hline LDL-c (mg /dL) & $100.10 \pm 29.85$ & $106.38 \pm 25.88$ & 0.48 \\
\hline TG (mg /dL) & $91.68 \pm 35.33$ & $114.28 \pm 27.9$ & 0.03 \\
\hline
\end{tabular}

G1: group of 6 meals per day; G2: group of 3 meals per day; BMI: body mass index; WC: waist circumference; FM: fat mass; RMR: resting metabolic rate; HOMA-IR: Homeostasis Model Assessment; QUICKI: Quantitative Insulin Sensitivity Check Index; TC: total cholesterol; LDL-c: low-density lipoprotein cholesterol; HDL-c: highdensity lipoprotein cholesterol; TG: triglycerides; SPAC: Scale of Periodic Alimentary Compulsion. Values are represented as mean \pm standard deviation and $95 \%$ confidence interval. Differences between groups analyzed by the t-test with significance when $\mathrm{p}<0.05$.

Table 2 details energy and macronutrient compositions of the weight loss diet programs. Table 3 shows the food intake habit and results related dietary intake during the intervention. Analyzing the groups at baseline, dietary consumption was similar. After intervention, in G1, there were a significant reduction in TEE (kcal) and total lipids (\%), while, protein intake (\%) increased. In G2, a decrease in TEE (\%) and carbohydrate (\%) was noted, as well as an increase in protein intake (\%). Considering the variation before and during the intervention between groups, G2 showed a greater reduction in carbohydrates consumption and a greater increase in protein consumption, compared to G1.

Table 2: Chemical composition (energy, macronutrients, fatty acids, fibers and cholesterol) of the prescribed diets (1400 to $2100 \mathrm{kcal} /$ day) in G1 and G2. 


\begin{tabular}{cccc}
\hline & G1 $(\mathbf{n}=\mathbf{1 9})$ & G2 $(\mathbf{n}=\mathbf{2 1})$ & $p$-value \\
\hline Energy (kcal) & $1521.05 \pm 118.2$ & $1552.38 \pm 188.73$ & 0.12 \\
\hline $\begin{array}{c}\text { Carbohydrates }(\% \\
\text { TEE) }\end{array}$ & $57.84 \pm 1.34$ & $58.01 \pm 1.39$ & 0.66 \\
\hline Proteins (\% TEE) & $20.33 \pm 0.73$ & & 0.82 \\
\hline Lipids (\% TEE) & $21.79 \pm 1.73$ & $20.01 \pm 0.87$ & 0.75 \\
\hline SFA (\% TEE) & $6.07 \pm 0.86$ & $21.91 \pm 1.83$ & 0.74 \\
\hline MUFA (\% TEE) & $7.86 \pm 2.79$ & $6.15 \pm 0.88$ & 0.76 \\
\hline PUFA (\% TEE) & $5.85 \pm 0.76$ & $7.81 \pm 2.85$ & 0.77 \\
\hline Total fibers (g) & $27.61 \pm 4.34$ & $5.85 \pm 0.80$ & 0.71 \\
\hline
\end{tabular}

G1: group of 6 meals per day; G2: group of 3 meals per day; SFA: saturated fatty acids; MUFA: monounsaturated fatty acids; PUFA: polyunsaturated fatty acids; TEE: total energy expenditure. Data are means \pm standard deviation Differences between groups analyzed by the t-test with significance when $p<0.05$.

Table 3: Comparison of dietary prescription and intake performed during the intervention between groups, and analysis of variations by group. 


\begin{tabular}{|c|c|c|c|c|c|}
\hline Baseline TEE (kcal) & $1952.40 \pm 571.36$ & \multirow{2}{*}{$-517.54 \pm 554.35$} & $1936.07 \pm 472.69$ & \multirow{2}{*}{$-551.95 \pm 497.65$} & 0.92 \\
\hline Final TEE (kcal) & $1434.86 \pm 205.28$ & & $1384.11 \pm 218.51$ & & 0.45 \\
\hline$p$-value ${ }^{*}$ & 0.01 & & $<0.001$ & & $0.83^{\#}$ \\
\hline Baseline CHO (\%) & $49.20 \pm 6.49$ & \multirow{2}{*}{$-0.24 \pm 5.55$} & $52.59 \pm 5.64$ & \multirow{2}{*}{$-5.89 \pm 6.05$} & 0.08 \\
\hline Final CHO (\%) & $48.95 \pm 5.18$ & & $46.70 \pm 5.63$ & & 0.19 \\
\hline$p$-value * & 0.84 & & $<0.001$ & & $<0.001^{\#}$ \\
\hline Baseline PTN (\%) & $18.05 \pm 4.19$ & \multirow{2}{*}{$3.33 \pm 4.72$} & $16.03 \pm 3.90$ & \multirow{2}{*}{$6.28 \pm 3.33$} & 0.12 \\
\hline Final PTN (\%) & $21.38 \pm 3.83$ & & $22.32 \pm 4.92$ & & 0.50 \\
\hline$p$-value $*$ & $<0.001$ & & $<0.001$ & & $0.03^{\#}$ \\
\hline Baseline LIP (\%) & $33.41 \pm 6.28$ & \multirow{2}{*}{$-3.50 \pm 6.62$} & $32.50 \pm 4.38$ & \multirow{2}{*}{$-1.30 \pm 6.23$} & 0.60 \\
\hline Final LIP (\%) & $29.91 \pm 5.39$ & & $31.20 \pm 5.86$ & & 0.47 \\
\hline$p$-value $*$ & 0.03 & & 0.34 & & $0.28^{\#}$ \\
\hline Baseline SFA (\%) & $10.67 \pm 2.41$ & \multirow{2}{*}{$-0.85 \pm 3.40$} & $10.37 \pm 1.88$ & \multirow{2}{*}{$-0.26 \pm 3.16$} & 0.66 \\
\hline Final SFA (\%) & $9.82 \pm 2.20$ & & $10.11 \pm 2.81$ & & 0.72 \\
\hline$p$-value ${ }^{*}$ & 0.29 & & 0.70 & & $0.57^{\#}$ \\
\hline Baseline MUFA (\%) & $9.17 \pm 2.35$ & \multirow{2}{*}{$-0.00 \pm 2.72$} & $9.49 \pm 2.08$ & \multirow{2}{*}{$-0.84 \pm 2.03$} & 0.65 \\
\hline Final MUFA (\%) & $9.16 \pm 1.96$ & & $8.64 \pm 1.98$ & & 0.41 \\
\hline$p$-value * & 0.99 & & 0.07 & & $0.28^{\#}$ \\
\hline Baseline PUFA (\%) & $7.22 \pm 3.31$ & \multirow{2}{*}{$-1.23 \pm 3.22$} & $6.47 \pm 1.74$ & \multirow{2}{*}{$-0.21 \pm 1.91$} & 0.38 \\
\hline Final PUFA (\%) & $5.99 \pm 1.37$ & & $6.26 \pm 2.03$ & & 0.62 \\
\hline$p$-value* & 0.11 & & 0.61 & & $0.22^{\#}$ \\
\hline Baseline TC (\%) & $332.24 \pm 142.34$ & \multirow{2}{*}{$23.93 \pm 184.29$} & $320.27 \pm 156.68$ & \multirow{2}{*}{$-30.76 \pm 171.02$} & 0.80 \\
\hline Final TC (\%) & $356.17 \pm 119.55$ & & $289.51 \pm 93.03$ & & 0.05 \\
\hline$p$-value * & 0.57 & & 0.41 & & $0.33^{\#}$ \\
\hline Baseline fiber (g) & $18.93 \pm 6.34$ & \multirow{2}{*}{$1.43 \pm 7.22$} & $18.89 \pm 8.03$ & \multirow{2}{*}{$-2.06 \pm 9.36$} & 0.98 \\
\hline Final fiber (g) & $20.36 \pm 5.56$ & & $16.83 \pm 5.81$ & & 0.05 \\
\hline$p$-value $*$ & 0.39 & & 0.32 & & $0.19^{\#}$ \\
\hline $\begin{array}{c}\text { Baseline number of } \\
\text { meals }\end{array}$ & $4.42 \pm 0.90$ & \multirow[t]{2}{*}{$1.31 \pm 0.82$} & $4.23 \pm 1.09$ & \multirow[t]{2}{*}{$-0.95 \pm 0.97$} & 0.56 \\
\hline Final number of meals & $5.73 \pm 0.45$ & & $3.28 \pm 0.56$ & & $<0.001$ \\
\hline$p$-value * & $<0.001$ & & $<0.001$ & & $<0.001^{\#}$ \\
\hline
\end{tabular}

G1: group of 6 meals per day; G2: group of 3 meals per day; TEE: total energy expenditure; CHO: carbohydrates; PTN: protein; LIP: lipids; SFA: saturated fatty acid; MUFA: monounsaturated fatty acid; PUFA: polyunsaturated fatty acid; TC: total cholesterol. Data are means \pm standard deviation. ${ }^{* *}$ Differences between groups assessed by the t-test. *Intragroup comparison (before and after intervention) by paired t- test. " Comparison of the difference in variables $(\Delta)$ calculated with the final and Baseline data.

At baseline, participants consumed an average of four meals a day in all groups (Figure 1). After intervention, G1 and G2 started to eat an average of six and three meals a day, respectively, statistically differing between groups (Figure 1). At baseline, G1 contained the highest percentage of participants having five and four meals per day and during the intervention they started to eat six 
meals (Figure 1). G2 showed the majority of participants eating four meals a day at baseline and the highest percentage changed to three daily meals after intervention (Figure 2).

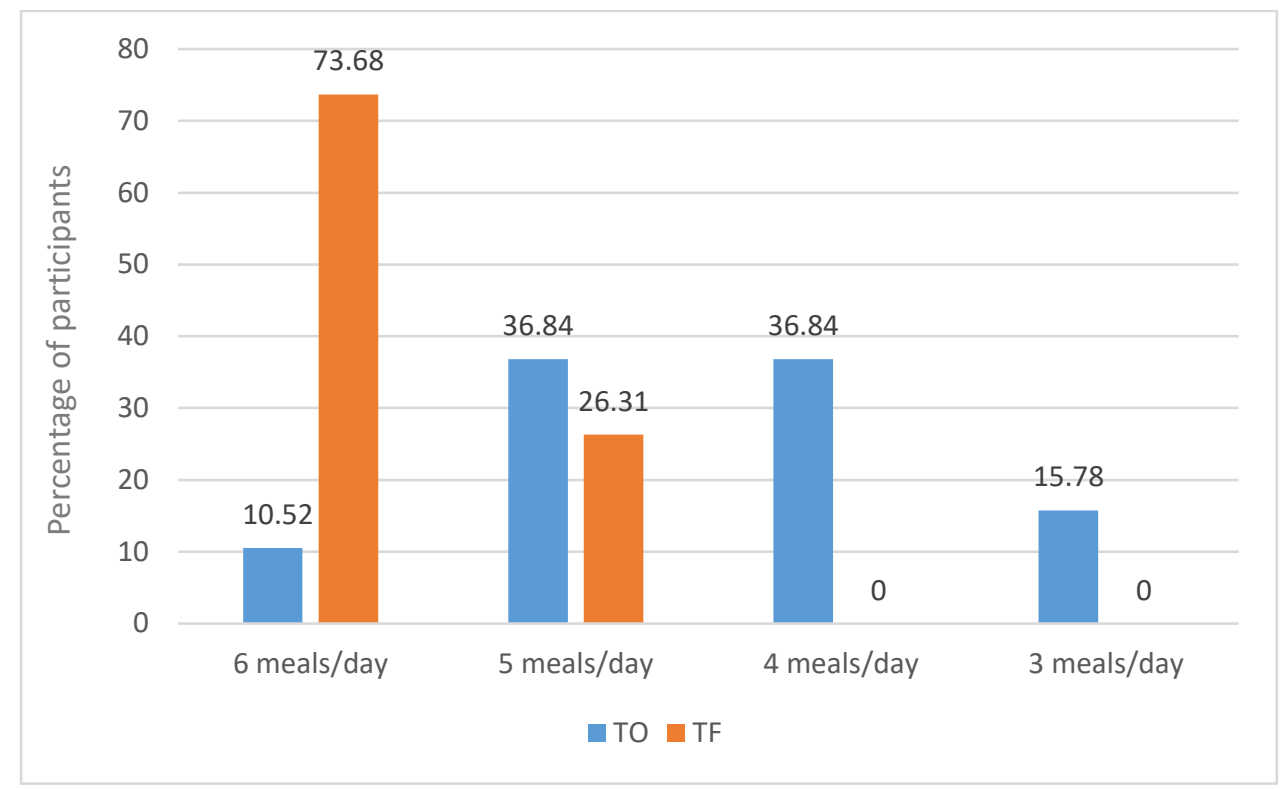

Figure 1. Food frequency at baseline and during intervention in G1.

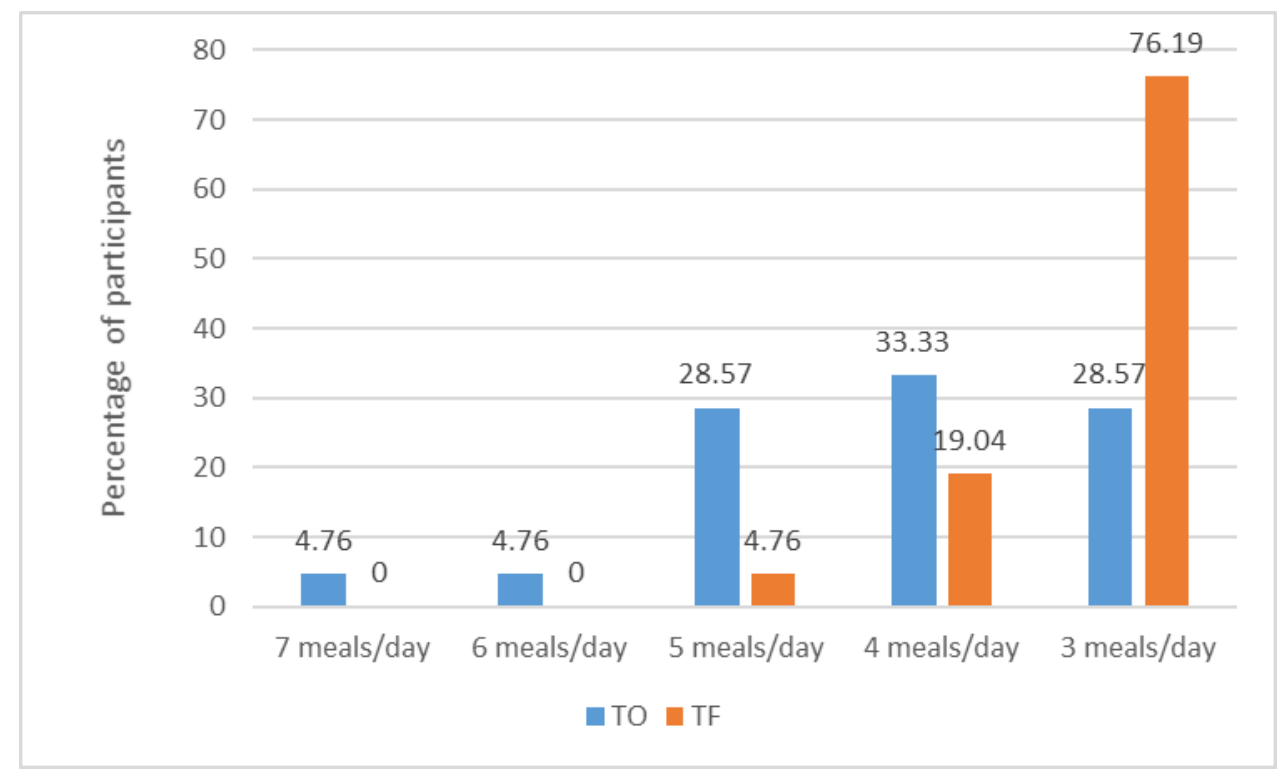

Figure 2. Food frequency at baseline and during intervention in G2.

Table 4 shows the self-reported acceptance by the participants. There was no significant difference between groups and, in addition, the percentage remained similar throughout the intervention period. No statistical difference was observed in eating behavior between groups in the baseline $(p=0.63)$ and final periods $(p=0.95)$. After intervention, the score obtained was lower than at the beginning of the research, differing statistically between groups and suggesting a reduction in episodes of food compulsion (G1 p=0.04; G2 p<0,01).

Table 4: Comparison of the percentage of acceptance in the returns of both groups.

$$
\text { G1 }(n=19) \quad \text { G2 }(n=21) \quad p \text {-value }
$$




\begin{tabular}{cccc}
\hline Acceptance T15 (\%) & $78.36 \pm 14.45$ & $70.19 \pm 15.76$ & 0.09 \\
\hline Acceptance T45 (\%) & $75.00 \pm 19.86$ & $70.09 \pm 19.60$ & 0.43 \\
\hline Acceptance T65 (\%) & $77.05 \pm 16.56$ & $72.14 \pm 14.96$ & 0.33 \\
\hline
\end{tabular}

G1: group of 6 meals per day; G2: group of 3 meals per day; T15: 15-day time; T45: 45-day time; T65: 65-day time. Differences between groups analyzed by the t-test with significance when $p<0.05$.

Data are means \pm standard deviation.

After intervention, there were no significant differences between and within groups for the variable RMR, substrate oxidation, respiratory coefficient (RC) (Table 5). The other laboratory and anthropometric variables did not differ between groups at baseline. After intervention, in intra-group comparison, it was observed that both groups significantly presented reduced body weight, BMI, WC, FM (kg), insulin, HOMA-IR (Tables 5 and 6). G1 also showed an increase in QUICKI (Table 6) and G2 showed a decrease in FM (\%) (Table 5) and TG (Table 6) and an increase in FFM (\%) (Table $5)$. There were no differences between the variations in anthropometric indicators between groups (Tables 5). Insulin levels and HOMA-IR decreased in both groups, although, even after reduction, these parameters remained higher in G2 (Table 6).

Table 5: Comparison of anthropometric indicators, body composition and EE between groups at baseline and after the intervention, and variations by group (mean $\pm \mathrm{SD})$.

$\begin{array}{lllll}\text { G1 }(\mathrm{n}=19) & \Delta & \mathrm{G} 2(\mathrm{n}=21) & \Delta & p \text { - value }^{* *}\end{array}$




\begin{tabular}{|c|c|c|c|c|c|}
\hline Baseline weight (kg) & $90.88 \pm 8.96$ & \multirow{2}{*}{$-2.99 \pm 3.35$} & $92.96 \pm 11.09$ & \multirow{2}{*}{$-3.60 \pm 3.50$} & 0.51 \\
\hline Final weight (kg) & $87.88 \pm 10.20$ & & $89.35 \pm 10.60$ & & 0.65 \\
\hline$p$-value * & $<0.001$ & & $<0.001$ & & $0.57^{\#}$ \\
\hline Baseline BMI $\left(\mathrm{kg} / \mathrm{m}^{2}\right)$ & $34.90 \pm 3.82$ & \multirow{2}{*}{$-1.16 \pm 1.32$} & $35.25 \pm 3.96$ & \multirow{2}{*}{$-1.35 \pm 1.30$} & 0.76 \\
\hline Final BMI $\left(\mathrm{kg} / \mathrm{m}^{2}\right)$ & $33.73 \pm 4.10$ & & $33.92 \pm 3.95$ & & 0.88 \\
\hline$p$-value * & $<0.001$ & & $<0.001$ & & $0.66^{\#}$ \\
\hline Baseline WC (cm) & $96.94 \pm 8.88$ & \multirow{2}{*}{$-4.27 \pm 3.64$} & $101.47 \pm 8.87$ & \multirow{2}{*}{$-4.36 \pm 4.00$} & 0.11 \\
\hline Final WC (cm) & $92.67 \pm 7.98$ & & $97.11 \pm 6.67$ & & 0.12 \\
\hline$p$-value * & $<0.001$ & & $<0.001$ & & $0.94^{\#}$ \\
\hline Baseline FFM (kg) & $48.88 \pm 4.10$ & \multirow{2}{*}{$-0.39 \pm 1.93$} & $49.05 \pm 5.17$ & \multirow{2}{*}{$-0.64 \pm 1.46$} & 0.91 \\
\hline Final FFM (kg) & $48.49 \pm 5.02$ & & $48.40 \pm 4.92$ & & 0.95 \\
\hline$p$-value $*$ & 0.38 & & 0.05 & & $0.65^{\#}$ \\
\hline Baseline FFM (\%) & $53.97 \pm 3.58$ & \multirow{2}{*}{$-0.91 \pm 10.26$} & $52.97 \pm 3.74$ & \multirow{2}{*}{$1.44 \pm 1.35$} & 0.39 \\
\hline Final FFM (\%) & $53.05 \pm 11.72$ & & $54.41 \pm 4.05$ & & 0.63 \\
\hline$p$-value ${ }^{*}$ & 0.70 & & $<0.001$ & & $0.33^{\#}$ \\
\hline Baseline FM (kg) & $41.99 \pm 6.45$ & \multirow{2}{*}{$-2.59 \pm 2.50$} & $43.90 \pm 7.65$ & \multirow{2}{*}{$-2.96 \pm 2.59$} & 0.39 \\
\hline Final FM (kg) & $39.40 \pm 7.19$ & & $40.94 \pm 7.55$ & & 0.51 \\
\hline$p$-value * & $<0.001$ & & $<0.001$ & & $0.64^{\#}$ \\
\hline Baseline FM (\%) & $46.02 \pm 3.58$ & \multirow{2}{*}{$0.91 \pm 10.26$} & $47.02 \pm 3.74$ & \multirow{2}{*}{$-1.44 \pm 1.35$} & 0.39 \\
\hline Final FM (\%) & $46.94 \pm 11.72$ & & $45.58 \pm 4.05$ & & 0.63 \\
\hline$p$-value * & 0.70 & & $<0.001$ & & $0.33^{\#}$ \\
\hline Baseline RMR (kcal) & $1467.10 \pm 121.08$ & \multirow{2}{*}{$-38.89 \pm 102.17$} & $1479.31 \pm 171.27$ & \multirow{2}{*}{$13.57 \pm 98.8$} & 0.79 \\
\hline Final RMR (kcal) & $1428.21 \pm 139.77$ & & $1492.88 \pm 172.08$ & & 0.19 \\
\hline$p$-value $*$ & 0.11 & & 0.53 & & $0.10^{\#}$ \\
\hline Baseline RC & $0.79 \pm 0.03$ & \multirow{2}{*}{$-0.008 \pm 0.05$} & $0.79 \pm 0.02$ & \multirow{2}{*}{$-0.003 \pm 0.04$} & 0.86 \\
\hline Final RC & $0.78 \pm 0.40$ & & $0.78 \pm 0.03$ & & 0.78 \\
\hline$p$-value * & 0.47 & & 0.71 & & $0.72^{\#}$ \\
\hline
\end{tabular}

G1: group of 6 meals per day; G2: group of 3 meals per day; BMI: body mass index; WC: waist circumference; FFM: fat-free mass; FM: fat mass; RMR: resting metabolic rate; RC: respiratory coefficient. Data are means \pm standard deviation. Differences between groups analyzed by the t-test with significance when $p<0.05$. Values are represented as mean \pm standard deviation ${ }^{* *}$ Differences between groups assessed by the $t$-test. ${ }^{*}$ Intragroup comparison (before and after intervention) by paired t- test. \# Comparison of the difference in variables $(\Delta)$ calculated with the final and baseline data.

Table 6: Comparison of laboratory variables between groups at baseline and after the intervention, and variations by group (mean $\pm \mathrm{SD})$.

\begin{tabular}{lllll}
$\mathrm{G} 1(\mathrm{n}=19)$ & $\Delta$ & $\mathrm{G} 2(\mathrm{n}=21)$ & $\Delta$ & $p$ - value ${ }^{* *}$ \\
\hline
\end{tabular}




\begin{tabular}{|c|c|c|c|c|c|}
\hline Baseline glucose (mg / dL) & $84.31 \pm 7.96$ & \multirow{2}{*}{$-1.05 \pm 5.88$} & $92.38 \pm 18.24$ & \multirow{2}{*}{$-4.19 \pm 10.46$} & 0.07 \\
\hline Final glucose (mg / dL) & $83.26 \pm 7.63$ & & $88.19 \pm 14.20$ & & 0.17 \\
\hline$p$-value ${ }^{*}$ & 0.44 & & 0.08 & & $0.24^{\#}$ \\
\hline Baseline insulin $(\mu \mathrm{U} / \mathrm{mL})$ & $17.63 \pm 8.06$ & \multirow{2}{*}{$-3.91 \pm 5.06$} & $24.54 \pm 12.39$ & \multirow{2}{*}{$-6.09 \pm 11.46$} & 0.04 \\
\hline Final insulin $(\mu \mathrm{U} / \mathrm{mL})$ & $13.72 \pm 5.88$ & & $18.44 \pm 7.00$ & & 0.02 \\
\hline$p$-value * & $<0.001$ & & 0.02 & & $0.43^{\#}$ \\
\hline Baseline HOMA-IR & $3.69 \pm 1.72$ & \multirow{2}{*}{$-0.84 \pm 1.22$} & $5.55 \pm 2.74$ & \multirow{2}{*}{$-1.48 \pm 2.58$} & 0.01 \\
\hline Final HOMA-IR & $2.84 \pm 1.31$ & & $4.06 \pm 1.79$ & & 0.01 \\
\hline$p$-value * & $<0.001$ & & 0.01 & & $0.31^{\#}$ \\
\hline Baseline QUICKI & $1.53 \pm 0.16$ & \multirow{2}{*}{$0.07 \pm 0.09$} & $1.51 \pm 0.29$ & \multirow{2}{*}{$-0.00 \pm 0.26$} & 0.78 \\
\hline Final QUICKI & $1.60 \pm 0.16$ & & $1.50 \pm 0.11$ & & 0.05 \\
\hline$p$-value * & $<0.001$ & & 0.97 & & $0.25^{\#}$ \\
\hline Baseline TC $(\mathrm{mg} / \mathrm{dL})$ & $169.52 \pm 35.49$ & \multirow{2}{*}{$-6.89 \pm 21.33$} & $178.38 \pm 28.52$ & $-10.42 \pm$ & 0.39 \\
\hline Final TC (mg/dL) & $162.63 \pm 32.85$ & & $167.95 \pm 24.08$ & 29.47 & 0.56 \\
\hline$p$-value $*$ & 0.17 & & 0.12 & & $0.66^{\#}$ \\
\hline Baseline HDL-c (mg/dL) & $51.21 \pm 13.06$ & \multirow{2}{*}{$-2.84 \pm 8.48$} & $49.09 \pm 10.58$ & \multirow{2}{*}{$-1.71 \pm 8.90$} & 0.58 \\
\hline Final HDL-c (mg/dL) & $48.36 \pm 10.58$ & & $47.38 \pm 12.53$ & & 0.78 \\
\hline$p$-value ${ }^{*}$ & 0.16 & & 0.38 & & $0.68^{\#}$ \\
\hline Baseline LDL-c (mg/dL) & $100.10 \pm 29.85$ & \multirow{2}{*}{$-2.47 \pm 14.70$} & $106.38 \pm 25.88$ & \multirow{2}{*}{$-5.52 \pm 25.71$} & 0.48 \\
\hline Final LDL-c (mg/dL) & $97.63 \pm 30.79$ & & $100.85 \pm 27.05$ & & 0.72 \\
\hline$p$-value * & 0.47 & & 0.33 & & $0.64^{\#}$ \\
\hline Baseline TG (mg/dL) & $91.68 \pm 35.33$ & \multirow{2}{*}{$-8.78 \pm 31.20$} & $114.28 \pm 27.9$ & $-15.57 \pm$ & 0.03 \\
\hline Final TG (mg/dL) & $82.89 \pm 32.05$ & & $98.71 \pm 28.15$ & 29.64 & 0.10 \\
\hline$p$-value * & 0.23 & & 0.02 & & $0.48^{\#}$ \\
\hline
\end{tabular}

G1: group of 6 meals per day; G2: group of 3 meals per day; BMI: body mass index; HOMA-IR: Homeostasis Model Assessment; QUICKI: Quantitative Insulin Sensitivity Check Index; TC: total cholesterol; LDL-c: lowdensity lipoprotein cholesterol; HDL-c: high-density lipoprotein cholesterol; TG: triglycerides. Data are means \pm standard deviation. Differences between groups analyzed by the t-test with significance when $\mathrm{p}<0.05$. Values are represented as mean \pm standard deviation ${ }^{* *}$ Differences between groups assessed by the t-test. ${ }^{*}$ Intragroup comparison (before and after intervention) by paired t- test. \# Comparison of the difference in variables $(\Delta)$ calculated with the final and baseline data.

There were no differences in the concentrations of fasting active ghrelin between groups, before and after the dietary intervention; however, the group with the lowest MF (G2) presented increased active ghrelin levels after the intervention (Table 7). Results of the correlation test showed no relationship in the baseline between concentration of active ghrelin levels with energy consumption $(r=-0.16, p=0.32)$, protein $(r=-0.07, p=0.68)$, glycidic $(r=0.06, p=0.71)$, lipidic $(r=-$ $0.03 \mathrm{p}=0.85)$ and PUFA $(\mathrm{r}=-0.01 \mathrm{p}=0.94)$ and with SPAC $(\mathrm{r}=-0.09, \mathrm{p}=0.58)$. Furthermore, active ghrelin levels after intervention were not related to loss of body weight $(r=-0.09, p=0.56)$, as well as there was no relationship between the behavior of ghrelin levels and loss of body weight $(r=-0.01, p$ $=0.93$ ).

Table 7: Comparison of active ghrelin levels between groups at baseline and after intervention and variations between groups, expressed as median (interquartile). 


\begin{tabular}{|c|c|c|c|c|c|}
\hline & G1 (n=16) & $\Delta$ & G2 $(n=20)$ & $\Delta$ & $p$-value $* *$ \\
\hline $\begin{array}{c}\text { Baseline active ghrelin } \\
\qquad(\mathrm{pg} / \mathrm{mL})\end{array}$ & $110.41(36.25 ; 155.06)$ & 5232 & $93.99(45.29 ; 151.88)$ & 240 & 0.86 \\
\hline $\begin{array}{l}\text { Final Active ghrelin } \\
\qquad(\mathrm{pg} / \mathrm{mL})\end{array}$ & $116.26(69.49 ; 264.08)$ & $(-135.79 ; 38.91)$ & $153.30(88.07 ; 319.76)$ & $(-234.27 ; 15.74)$ & 0.51 \\
\hline$p$-value * & 0.13 & & 0.03 & & $0.74^{\#}$ \\
\hline
\end{tabular}

G1: group of 6 meals per day; G2: group of 3 meals per day. Data are in median (interquartile). Significant differences when $\mathrm{p}<0.05$. ${ }^{* *}$ Differences between groups assessed by the Mann Whitney test. ${ }^{*}$ Intra-group comparison using the Wilcoxon test. \# Comparison of the difference in variables $(\Delta)$ calculated with the final and baseline data.

\section{Discussion}

Effects of MF in the population with obesity is still controversial and it seems that changing frequency in calorie-restricted eating plans may not affect weight and body composition [8,11].

In the present study, data on dietary intake, including the number of daily meals and self-report of acceptance, demonstrated that women with obesity had satisfactory adherence to the diet plan and the proposed MF, with no difference between groups. A controlled and randomized research carried out in women with obesity and glucose intolerance or type 2 diabetes mellitus, evaluated the result of low-calorie diet with different MF (six and three meals a day), for 12 weeks, and suggested that more MF may be useful for individuals who are not motivated to follow calorie restricted diets [37].

Authors should discuss the results and how they can be interpreted in perspective of previous studies and of the working hypotheses. The findings and their implications should be discussed in the broadest context possible. Future research directions may also be highlighted.

In our study, the different MF did not influence anthropometric indicators, since both groups reduced body weight, WC and BMI, with no difference between them. Likewise, was observed in a study conducted including 47 adult women with obesity, randomized into two groups (hypocaloric diet, three or six meals per day), for three months. They showed no differences in weight, WC and BMI between groups [38]. In another study, it was reported that in individuals with obesity, lower MF (three meals per day versus five meals per day) provided greater reduction in body weight, assessed over a three-week period, however, the authors suggested further investigations [39].

In the present study, the lower MF provided an increase in FFM (\%), in addition to a reduction in FM (kg). Our results differed from a clinical trial including women with obesity, randomized into two groups (two and six meals a day - low calorie diet with controlled portions), for two weeks. They verified that FFM (\%) increased in the highest MF, suggesting that eating frequency can preserve the FFM during weight loss [40]. In another study, 16 adults with obesity were randomized into two groups (three and six meals per day) and subjected to the same energy restriction for eight weeks, and the results showed similar weight reduction, FM $(\mathrm{kg})$ and FFM $(\mathrm{kg})$ between the groups, so increasing the MF does not promote greater loss of body weight [9].

MF is cited as a factor that can interfere in EE in humans, since it can alter the TEF and consequently the body weight [41]. Le Blanc et al. (1993) [42] showed that consuming four small meals, at 40-minute intervals, compared to a large one, led to an increase in postprandial thermogenesis, caused by lipid mobilization resulting from repeated stimulation of the sympathetic nervous system by foods containing fats in six eutrophic adults. In the present study, consuming more times during the day did not promote an increase in EE, therefore, despite changes in body composition, RMR was sustained before and after dietary intervention in the groups analyzed, in women with obesity.

It is reported that the smaller MF (three/day), in normal weight, decreases serum glucose concentrations throughout the day, indicating improvement in the glycemic profile, as well as 
promoting peaks of postprandial insulin secretion [43]. In the present study, all groups had normal blood glucose and IR values. MF did not promote alterations in fasting glucose in both groups. Insulin and HOMA-IR were higher in G2 at the beginning of the study and remained the same, with no differences between groups, although G1 improved insulin sensitivity. In both MF there was a reduction in IR and insulinemia. A randomized, crossover study conducted for 24 weeks including women with overweight and polycystic ovary syndrome showed that six, compared to three meals for day, improved insulin sensitivity after the oral glucose tolerance test and did not differ in glycaemia, lipemia and glycated hemoglobin [37]. A current study showed that TG was the only lipid fraction that reduced with the lowest MF, likewise the consumption of carbohydrate (\%) was lower in the group in question, which may have favored this result, in addition, another hypothesis is the possibility of higher volume of meals increase postprandial insulin and stimulating lipoprotein lipase from adipose tissue to hydrolyze TG in non-esterified fatty acid and glycerol to be stored [44].

In the present study, no difference was observed in the plasma concentration of fasting active ghrelin before and after the intervention with a low-calorie diet between the groups. In contrast, the group with the lowest MF increased ghrelin secretion after dietary intervention. We emphasize that in our study, both groups had similar weight loss and energy consumption and it seems that these factors are more related to changes in ghrelin than the MF itself during the day.

A research analyzed the effect of the hypocaloric diet with different MF (two and six meals a day), in 54 overweight and obese women, found that the lower MF led to a greater reduction in body weight and increased the active ghrelin secretion [14]. The elevation of fasting active ghrelin can be considered a beneficial factor for stimulating hunger in the morning, since omission of breakfast is associated with an increased risk of glycemic dysregulation and visceral adiposity. In addition, this increase may have occurred in the lower MF due to the longer fasting time between meals [14,45,46]. Still, the increase in active ghrelin in the long term can cause difficulty in adhering to dietary treatment, since it is an orexigenic hormone [47].

It is known that the food composition is able to change the secretion of total ghrelin. In normal weight individuals, observed after ingestion of three test meals composed of $80 \%$ of the analyzed macronutrients, carbohydrates and proteins were more effective in suppressing active or total orexigenic hormone [48]. In the present study, we found no relationship between lipid consumption and ghrelin concentration at baseline, however, lipid consumption did not differ between groups, which could explain the non-relationship with active hormone secretion. It should also be noted that our prescription was of a hypocaloric and normolipidic diet and the study conducted by FosterSchubert et al (2008) used a test meal with a lipid load to assess ghrelin secretion. In eutrophic patients, it is pointed out that the intake of a diet rich in PUFA for seven days led to a reduction in total fasting ghrelin secretion [49].

Unlike the aforementioned study, our prescription maintained the normal distribution of lipid fractions in the diet, not using a high PUFA diet. The higher consumption of carbohydrates and total lipids cannot alter the secretion of ghrelin in overweight and obese men [50]. In the present study, we did not observe a relationship between fasting active ghrelin and protein intake, carbohydrate and PUFA. We emphasize that the nutrients evaluated showed similar consumption between groups, without using loads, which may include in the absence of a relationship with ghrelin.

\section{Conclusions}

The consumption of hypocaloric diets with different frequencies for 12 weeks can reduce body weight, BMI, WC, SPAC, FM (kg), insulin and HOMA-IR. Regardless of MF, the diet moderately reduced in energy promoted a decrease in weight and an improvement in the body composition.

Smaller number of meals eaten per day seems to have better results in triglyceridemia and in the increase of FFM (\%) in women with obesity. However, it is suggestive that the greater number of meals throughout the day may favor an increase in insulin sensitivity.

Decreased MF led to an elevation in active ghrelin levels, however, further studies are needed to assess the influence of MF on the secretion of this hormone and its impact on food intake and body composition and their effects on plasma lipids. 
Both groups showed changes in MF, followed the proposed dietary prescription and intervals between meals, therefore, MF resulted in satisfactory adherence to the hypocaloric diet. G1 and G2 decreased energy intake and increased protein intake, however, G1 reduced the consumption of total lipids (\%) and increased the number of meals, while in G2 there was a lower carbohydrate intake (\%) and number of meals.

The different MF were not able to alter the EE and the oxidation of substrates, despite the reduction in body mass in both groups and the increase in FFM (\%) in G2, the RMR remained constant. In addition, moderate hypocaloric diets were able to influence glucose metabolism, reducing insulin and its resistance.

Considering there are few clinical trials evaluating the effect of MF on body composition, blood glucose, lipemia, active ghrelin and EE in individuals with obesity and the heterogeneity of the results indicated in the research, it is relevant that more controlled studies be conducted in order to point out new possibilities that improve dietary treatment in obesity.

\section{Limitations of the study}

Among the limitations of the present study, the small sample size can be mentioned; In addition, the intervention time was 12 weeks and cannot affirm the possible long-term effects of MF, however, the sample was homogeneous, including participants of childbearing age, same sex, with similarity in the BMI classification and without comorbidities, which minimizes the metabolic differences.

Author Contributions: Conceptualization, ERL and EDG; Formal analysis, ERL, MSP and EDG; Investigation, EDG, ERL, MST, LOS and MSP; Methodology, ELR; Project administration, EDG; Resources, ELR; Supervision, ELD; Writing - original draft, EDG and ELR; Writing - review \& editing, ERL, EDG, MSP, MST, LOS and HMP.

Funding: This research was funded by Conselho Nacional de Desenvolvimento Científico (CNPq), grant number 432339/2016-7.

Acknowledgments: To the Laboratório de Análises Clínicas da Faculdade de Farmácia (LACFAR) and Laboratório de Avaliação Nutricional (LANUTRI) for their support and partnership.

Conflicts of Interest: The authors declare no conflict of interest.

\section{References}

1. Pi-Sunyer, F. X. The Obesity Epidemic: Pathophysiology and Consequences of Obesity. Obes.

Res. 2002, 10 Suppl 2, 97S-104S. https://doi.org/10.1038/oby.2002.202.

2. WHO. Obesity and overweight, 2016. https://www.who.int/news-room/fact-sheets/detail/obesityand-overweight (accessed Aug 25, 2018).

3. Salaün, H.; Thariat, J.; Vignot, M.; Merrouche, Y.; Vignot, S. [Obesity and cancer]. Bull. Cancer (Paris) 2017, 104 (1), 30-41. https://doi.org/10.1016/j.bulcan.2016.11.012.

4. ABESO. Diretrizes Brasileiras de Obesidade, 2016. http://www.abeso.org.br/diretrizes (accessed Oct 19, 2019).

5. Adults (US), N. O. E. I. E. P. on the I., Evaluation, and Treatment of Obesity in. Clinical Guidelines on the Identification, Evaluation, and Treatment of Overweight and Obesity in Adults; National Heart, Lung, and Blood Institute, 1998.

6. Raynor, H. A.; Champagne, C. M. Position of the Academy of Nutrition and Dietetics: Interventions for the Treatment of Overweight and Obesity in Adults. J. Acad. Nutr. Diet. 2016, 116 (1), 129-147. https://doi.org/10.1016/j.jand.2015.10.031. 
7. Seagle, H. M.; Strain, G. W.; Makris, A.; Reeves, R. S.; American Dietetic Association. Position of the American Dietetic Association: Weight Management. J. Am. Diet. Assoc. 2009, 109 (2), 330-346. https://doi.org/10.1016/j.jada.2008.11.041.

8. Hutchison, A. T.; Heilbronn, L. K. Metabolic Impacts of Altering Meal Frequency and Timing Does When We Eat Matter? Biochimie 2016, 124, 187-197. https://doi.org/10.1016/j.biochi.2015.07.025.

9. Cameron, J. D.; Cyr, M.-J.; Doucet, E. Increased Meal Frequency Does Not Promote Greater Weight Loss in Subjects Who Were Prescribed an 8-Week Equi-Energetic Energy-Restricted Diet. Br. J. Nutr. 2010, 103 (8), 1098-1101. https://doi.org/10.1017/S0007114509992984.

10. Megson, M.; Wing, R.; Leahey, T. M. Effects of Breakfast Eating and Eating Frequency on Body Mass Index and Weight Loss Outcomes in Adults Enrolled in an Obesity Treatment Program. J. Behav. Med. 2017, 40 (4), 595-601. https://doi.org/10.1007/s10865-017-9828-0.

11. Varady, K. A. Meal Frequency and Timing: Impact on Metabolic Disease Risk. Curr. Opin. Endocrinol. Diabetes Obes. 2016, 23 (5), 379-383. https://doi.org/10.1097/MED.0000000000000280. 12. Smeets, A. J.; Westerterp-Plantenga, M. S. Acute Effects on Metabolism and Appetite Profile of One Meal Difference in the Lower Range of Meal Frequency. Br. J. Nutr. 2008, 99 (6), 1316-1321. https://doi.org/10.1017/S0007114507877646.

13. Kulovitz, M. G.; Kravitz, L. R.; Mermier, C.; Gibson, A. L.; Conn, C. A.; Kolkmeyer, D.; Kerksick, C. M. Potential Role of Meal Frequency as a Strategy for Weight Loss and Health in Overweight or Obese Adults. Nutr. Burbank Los Angel. Cty. Calif 2014, 30 (4), 386-392.

https://doi.org/10.1016/j.nut.2013.08.009.

14. Belinova, L.; Kahleova, H.; Malinska, H.; Topolcan, O.; Windrichova, J.; Oliyarnyk, O.; Kazdova, L.; Hill, M.; Pelikanova, T. The Effect of Meal Frequency in a Reduced-Energy Regimen on the Gastrointestinal and Appetite Hormones in Patients with Type 2 Diabetes: A Randomised Crossover Study. PloS One 2017, 12 (4), e0174820. https://doi.org/10.1371/journal.pone.0174820. 15. Cummings, D. E.; Weigle, D. S.; Frayo, R. S.; Breen, P. A.; Ma, M. K.; Dellinger, E. P.; Purnell, J. Q. Plasma Ghrelin Levels after Diet-Induced Weight Loss or Gastric Bypass Surgery. N. Engl. J. Med. 2002, 346 (21), 1623-1630. https://doi.org/10.1056/NEJMoa012908.

16. FAO/WHO/UNU. Human energy requirements. Joint FAO/WHO/UNU Expert Consultation. Roma, 2001. https://www.who.int/nutrition/publications/nutrientrequirements/9251052123/en/ (accessed Nov 19, 2019).

17. IOM. Dietary reference intakes for energy, carbohydrate, fiber, fat, fatty acids, cholesterol, protein and amino acids. Washington (DC): National Academy Press, 2005.

18. FAO. Fats and fatty acids in human nutrition. Volume 91 FAO; Rome, Italy:2010.

19. WHO. Diet, Nutrition, and the Prevention of Chronic Diseases: Report of a WHO-FAO Expert Consultation; Geneva, 2003.

20. Yokoo, E. M.; Pereira, R. A.; Veiga, G. V. da; Nascimento, S.; Costa, R. S.; Marins, V. M. R. de; Lobato, J. C. P.; Sichieri, R. Proposta metodológica para o módulo de consumo alimentar pessoal na pesquisa brasileira de orçamentos familiares. Rev. Nutr. 2008, 21 (6), 767-776.

https://doi.org/10.1590/S1415-52732008000600015.

21. Matsudo, S.; Araújo, T.; Matsudo, V.; Andrade, D.; Andrade, E.; Oliveira, L. C.; Braggion, G. QUESTIONÁRIO INTERNACIONAL DE ATIVIDADE FÍSICA (IPAQ): ESTUPO DE VALIDADE E 
REPRODUTIBILIDADE NO BRASIL. Rev. Bras. Atividade Física Saúde 2001, 6 (2), 5-18. https://doi.org/10.12820/rbafs.v.6n2p5-18. 22. Blundell, J. E.; Gillett, A. Control of Food Intake in the Obese. Obes. Res. 2001, 9 Suppl 4, 263S270S. https://doi.org/10.1038/oby.2001.129.

23. Freitas, S.; Lopes, C. S.; Coutinho, W.; Appolinario, J. C. Tradução e adaptação para o português da Escala de Compulsão Alimentar Periódica. Braz. J. Psychiatry 2001, 23 (4), 215-220. https://doi.org/10.1590/S1516-44462001000400008.

24. WHO. Obesity: preventing and managing the global epidemic, 2000. http://www.who.int/entity/nutrition/publications/obesity/WHO_TRS_894/en/index.html (accessed Aug 25, 2018).

25. Lukaski, H. C.; Johnson, P. E.; Bolonchuk, W. W.; Lykken, G. I. Assessment of Fat-Free Mass Using Bioelectrical Impedance Measurements of the Human Body. Am. J. Clin. Nutr. 1985, 41 (4), 810-817. https://doi.org/10.1093/ajcn/41.4.810.

26. Casas-Agustench, P.; López-Uriarte, P.; Bulló, M.; Ros, E.; Gómez-Flores, A.; Salas-Salvadó, J. Acute Effects of Three High-Fat Meals with Different Fat Saturations on Energy Expenditure, Substrate Oxidation and Satiety. Clin. Nutr. Edinb. Scotl. 2009, 28 (1), 39-45. https://doi.org/10.1016/j.clnu.2008.10.008.

27. Kyle, U. G.; Bosaeus, I.; De Lorenzo, A. D.; Deurenberg, P.; Elia, M.; Manuel Gómez, J.; Lilienthal Heitmann, B.; Kent-Smith, L.; Melchior, J.-C.; Pirlich, M.; Scharfetter, H.; M W J Schols, A.; Pichard, C.; ESPEN. Bioelectrical Impedance Analysis-Part II: Utilization in Clinical Practice. Clin. Nutr. Edinb. Scotl. 2004, 23 (6), 1430-1453. https://doi.org/10.1016/j.clnu.2004.09.012.

28. Sampaio, H. A. de C. Aspectos nutricionais relacionados ao ciclo menstrual. Rev. Nutr. 2002, 15 (3), 309-317. https://doi.org/10.1590/S1415-52732002000300007.

29. Weir, J. B. D. B. New Methods for Calculating Metabolic Rate with Special Reference to Protein Metabolism. J. Physiol. 1949, 109 (1-2), 1-9. https://doi.org/10.1113/jphysiol.1949.sp004363.

30. Kostner, G. M.; Avogaro, P.; Bon, G. B.; Cazzolato, G.; Quinci, G. B. Determination of HighDensity Lipoproteins: Screening Methods Compared. Clin. Chem. 1979, 25 (6), 939-942.

31. Lott, J. A.; Turner, K. Evaluation of Trinder's Glucose Oxidase Method for Measuring Glucose in Serum and Urine. Clin. Chem. 1975, 21 (12), 1754-1760.

32. McGowan, M. W.; Artiss, J. D.; Strandbergh, D. R.; Zak, B. A Peroxidase-Coupled Method for the Colorimetric Determination of Serum Triglycerides. Clin. Chem. 1983, 29 (3), 538-542.

33. Richmond, W. Preparation and Properties of a Cholesterol Oxidase from Nocardia Sp. and Its Application to the Enzymatic Assay of Total Cholesterol in Serum. Clin. Chem. 1973, 19 (12), 13501356.

34. Friedwald, W.; Levy, R.; Fredrickson, D. Estimation of the Concentration of Low-Density Lipoprotein Cholesterol in Plasma, Without Use of the Preparative Ultracentrifuge I Clinical Chemistry. 1972, pp 499-502.

35. Matthews, D. R.; Hosker, J. P.; Rudenski, A. S.; Naylor, B. A.; Treacher, D. F.; Turner, R. C. Homeostasis Model Assessment: Insulin Resistance and Beta-Cell Function from Fasting Plasma Glucose and Insulin Concentrations in Man. Diabetologia 1985, 28 (7), 412-419. https://doi.org/10.1007/bf00280883. 
36. Katz, A.; Nambi, S. S.; Mather, K.; Baron, A. D.; Follmann, D. A.; Sullivan, G.; Quon, M. J. Quantitative Insulin Sensitivity Check Index: A Simple, Accurate Method for Assessing Insulin Sensitivity in Humans. J. Clin. Endocrinol. Metab. 2000, 85 (7), 2402-2410.

https://doi.org/10.1210/jcem.85.7.6661.

37. Papakonstantinou, E.; Kontogianni, M. D.; Mitrou, P.; Magriplis, E.; Vassiliadi, D.; Nomikos, T.; Lambadiari, V.; Georgousopoulou, E.; Dimitriadis, G. Effects of 6 vs 3 Eucaloric Meal Patterns on Glycaemic Control and Satiety in People with Impaired Glucose Tolerance or Overt Type 2 Diabetes: A Randomized Trial. Diabetes Metab. 2018, 44 (3), 226-234. https://doi.org/10.1016/j.diabet.2018.03.008.

38. Yildiran, Hi.; Mercanligil, S. M. Does Increasing Meal Frequency Improve Weight Loss and Some Biochemical Parameters in Overweight/Obese Females? Nutr. Hosp. 2019, 36 (1), 66-72. https://doi.org/10.20960/nh.2191.

39. Garrow, J. S.; Durrant, M.; Blaza, S.; Wilkins, D.; Royston, P.; Sunkin, S. The Effect of Meal Frequency and Protein Concentration on the Composition of the Weight Lost by Obese Subjects. Br. J. Nutr. 1981, 45 (1), 5-15. https://doi.org/10.1079/bjn19810072.

40. Alencar, M. K.; Beam, J. R.; McCormick, J. J.; White, A. C.; Salgado, R. M.; Kravitz, L. R.; Mermier, C. M.; Gibson, A. L.; Conn, C. A.; Kolkmeyer, D.; Ferraro, R. T.; Kerksick, C. M. Increased Meal Frequency Attenuates Fat-Free Mass Losses and Some Markers of Health Status with a Portion-Controlled Weight Loss Diet. Nutr. Res. N. Y. N 2015, 35 (5), 375-383. https://doi.org/10.1016/j.nutres.2015.03.003.

41. Belko, A. Z.; Barbieri, T. F. Effect of Meal Size and Frequency on the Thermic Effect of Food. Nutr. Res. 1987, 7 (3), 237-242. https://doi.org/10.1016/S0271-5317(87)80013-1.

42. LeBlanc, J.; Mercier, I.; Nadeau, A. Components of Postprandial Thermogenesis in Relation to Meal Frequency in Humans. Can. J. Physiol. Pharmacol. 1993, 71 (12), 879-883. https://doi.org/10.1139/y93-133.

43. Munsters, M. J. M.; Saris, W. H. M. Effects of Meal Frequency on Metabolic Profiles and Substrate Partitioning in Lean Healthy Males. PLoS ONE 2012, 7 (6).

https://doi.org/10.1371/journal.pone.0038632.

44. Yazıc1, D.; Sezer, H. Insulin Resistance, Obesity and Lipotoxicity. Adv. Exp. Med. Biol. 2017, 960, 277-304. https://doi.org/10.1007/978-3-319-48382-5_12.

45. Mekary, R. A.; Giovannucci, E.; Willett, W. C.; van Dam, R. M.; Hu, F. B. Eating Patterns and Type 2 Diabetes Risk in Men: Breakfast Omission, Eating Frequency, and Snacking. Am. J. Clin. Nutr. 2012, 95 (5), 1182-1189. https://doi.org/10.3945/ajcn.111.028209.

46. Reutrakul, S.; Hood, M. M.; Crowley, S. J.; Morgan, M. K.; Teodori, M.; Knutson, K. L. The Relationship between Breakfast Skipping, Chronotype, and Glycemic Control in Type 2 Diabetes. Chronobiol. Int. 2014, 31 (1), 64-71. https://doi.org/10.3109/07420528.2013.821614.

47. MAKRIS, C. M.; ALEXANDROU, A.; PAPATSOUTSOS, G. E.; MALIETZIS, G.; TSILIMIGRAS, I. D.; GUERRON, D. A.; MORIS, D. Ghrelin and Obesity: Identifying Gaps and Dispelling Myths. A Reappraisal. In Vivo 2017, 31 (6), 1047-1050. https://doi.org/10.21873/invivo.11168.

48. Foster-Schubert, K. E.; Overduin, J.; Prudom, C. E.; Liu, J.; Callahan, H. S.; Gaylinn, B. D.; Thorner, M. O.; Cummings, D. E. Acyl and Total Ghrelin Are Suppressed Strongly by Ingested 
Proteins, Weakly by Lipids, and Biphasically by Carbohydrates. J. Clin. Endocrinol. Metab. 2008, 93 (5), 1971-1979. https://doi.org/10.1210/jc.2007-2289.

49. Stevenson, J. L.; Paton, C. M.; Cooper, J. A. Hunger and Satiety Responses to High-Fat Meals after a High-Polyunsaturated Fat Diet: A Randomized Trial. Nutr. Burbank Los Angel. Cty. Calif 2017, 41, 14-23. https://doi.org/10.1016/j.nut.2017.03.008.

50. Adamska-Patruno, E.; Ostrowska, L.; Goscik, J.; Pietraszewska, B.; Kretowski, A.; Gorska, M. The Relationship between the Leptin/Ghrelin Ratio and Meals with Various Macronutrient Contents in Men with Different Nutritional Status: A Randomized Crossover Study. Nutr. J. 2018, 17 (1), 118. https://doi.org/10.1186/s12937-018-0427-x. 